\title{
Editorial
}

\section{Switched Dynamics with Its Applications}

\author{
Chang-Hua Lien, ${ }^{1}$ Lixian Zhang, ${ }^{2}$ Sundarapandian Vaidyanathan, ${ }^{3}$ \\ and Hamid Reza Karimi ${ }^{4}$ \\ ${ }^{1}$ Department of Marine Engineering, National Kaohsiung Marine University, Kaohsiung 811, Taiwan \\ ${ }^{2}$ Research Institute of Intelligent Control and Systems, Harbin Institute of Technology, P.O. Box 3015, Yikuang Street No. 2, \\ Nangang District, Harbin 150080, China \\ ${ }^{3}$ Research and Development Centre, Vel Tech University, No. 42 Avadi-Vel Tech Road, Avadi, Chennai, Tamil Nadu 600062, India \\ ${ }^{4}$ Department of Engineering, Faculty of Engineering and Science, University of Agder, 4898 Grimstad, Norway
}

Correspondence should be addressed to Chang-Hua Lien; chlien@mail.nkmu.edu.tw

Received 25 November 2013; Accepted 25 November 2013; Published 10 February 2014

Copyright (C) 2014 Chang-Hua Lien et al. This is an open access article distributed under the Creative Commons Attribution License, which permits unrestricted use, distribution, and reproduction in any medium, provided the original work is properly cited.

In recent years, switched dynamics has attracted a rapidly growing attention in the fields of networked control systems, sliding mode control systems, switched systems, and T$\mathrm{S}$ fuzzy systems. This special issue has a strong impact on many real-world systems, like automated highway systems, automotive engine control system, chemical process, constrained robotics, network systems, power systems and power electronics, robot manufacture, stepper motors, and even water quality control. Systems with switching may produce many complicated nonlinear system behaviors, such as multiple limit cycles and chaos. Nowadays, the analysis of switched dynamics of nonlinear systems still possesses many new challenges to researchers. When studying switched dynamics of practical systems, it is very crucial to be able to completely characterize the dynamical properties of the nonlinear systems with switching. This special issue aims at gathering research works focusing on the developments and discoveries of switched dynamics with its applications.

This special issue contains twenty papers, the contents of which are summarized as follows.

"Sliding mode control with state derivative output feedback in reciprocal state space form" by Y.-W. Tseng and Y.-N. Wang considers the design of novel sliding mode control by state derivative output feedback in nontraditional reciprocal state space (RSS) form. Novel switching function and approaching condition based on the derivative of sliding surface are introduced. A sufficient condition is developed to find the upper bound of system uncertainty and guarantee the stability for sliding surface in robustness analysis.

"A prediction-correction dynamic method for large-scale generalized eigenvalue problems" by X.-L. Luo et al. presents a new prediction-correction method of differential-algebraic equations for the smallest generalized eigenvalue problem. The smallest generalized eigenvalue problem is converted into an equivalent constrained optimization problem. According to the Karush-Kuhn-Tucker conditions of this special equality-constrained problem, a special continuous dynamical system of differential-algebraic equations is obtained. A prediction-correction method is constructed to follow the differential-algebraic equations.

"Extension sliding mode controller for maximum power point tracking of hydrogen fuel cells" by M.-H. Wang et al. proposes a maximum power point tracking (MPPT) control scheme based on extension theory to stabilize the output of a fuel cell at the point of maximum power. The simulation results confirm the ability of the controller to stabilize the output power at the maximum power point despite sudden changes in the temperature, hydrogen pressure, and membrane water content. The transient response time of the proposed controller is shown to be faster than that of existing sliding mode and extremum seeking controllers.

"Controller design of multi-input multioutput time-delay large-scale system" by C.-W. Lin et al. provides a novel feedback linearization controller of nonlinear multi-input 
multi-utput time-delay large-scale systems to obtain both the tracking and almost disturbance decoupling (ADD) performances. The significant contribution is to build up a control law such that the overall closed-loop system is stable for given initial condition and bounded tracking trajectory.

"Optimal control for a family of systems in novel state derivative space form with experiment in a double inverted pendulum system" by Y.-W. Tseng and J.-G. Hsieh proposes an optimal control for a family of systems in novel state derivative space (SDS) form. This optimal state derivative feedback may be implemented by the equivalent state feedback. The LQR design for a double inverted pendulum system is implemented to illustrate the use of the proposed method.

"Applications of fuzzy sliding mode control for a gyroscope system" by S.-C. Chen et al. presents the application of the fuzzy sliding mode for a gyroscope system status control. The state response analysis of the gyroscope system revealed highly nonlinear and chaotic subharmonic motions of $2 \mathrm{~T}$ during state formation. The current study discussed the use of tracking control in the sliding mode control and fuzzy sliding mode control of a gyroscope control system. The gyroscope system drives from chaotic motion to periodic motion.

"A dynamic cooperative scheme with multiple antennas for indoor mobile robot localization" by C.-L. Chang and B.$\mathrm{H}$. Wu develops a spatial cooperative diversity and decisionmaking technique to enhance signal detection and indoor mobile robot positioning performance of a global positioning satellite system receiver. A signal processing experimental platform is also established to receive actually indoor GNSS signals for verification. The proposed scheme is capable of effectively promoting the postcorrelation signal-to-noise ratio (SNR) capability of a GNSS receiver under the indoor environment.

"Multiple input delays estimation using an artificial bee colony algorithm" by W.-D. Chang considers the time delay estimation of discrete-time state equation with multiple input delays. A new artificial bee colony (ABC) algorithm is utilized to estimate the input delays. Based on the proposed algorithm, the unknown system input delays can be solved by minimizing the quadratic cost function of the system.

"Research on RBV control strategy of large angle maneuver" by J. Xu et al. presents a nonlinear flight control design based on neural network robust adaptive dynamic inversion for a class of reusable launch vehicles (RBV). The nonlinear characteristics of aerodynamic coefficients varying with mach numbers, attack angle, and sideslip angle are used such that the complete nonlinear 6-DOF model of RBV is established. The nonlinear dynamic inversion control strategy is then developed to achieve the pseudolinear decoupling of RBV. The external disturbance and network approximating error are suppressed by the used robust adaptive control. Lyapunov stability theories show that all error signals of closed-loop system are uniformly bounded finally under the control strategy.

"Robust coordinated formation for multiple surface vessels based on backstepping sliding mode control" by $\mathrm{M}$. Fu et al. investigates the problem of coordinated formation control for multiple surface vessels in the presence of unknown external disturbances. To achieve the robustness against unknown external disturbances, a new robust leaderless coordinated formation control algorithm based on backstepping sliding mode control is proposed. Furthermore, the adaptive control law proposed to estimate unknown disturbances is also extended to obtain the robust coordinated formation control algorithm.

"Data acquisition and transmission system for building energy consumption monitoring" by L. Zhao et al. presents a low-cost and small-sized collector design based on the STM32 microcontroller, which can be placed in building easily to implement the work of data acquisition, store, and transmission. The collector gathers the electricity, water, heat, and so forth, energy consumption data through the RS485 field bus, stores the data into SD card with mass storage, and finally, uses internet to finish communication and transmission to data server though TCP protocol.

"Observer-based robust $H_{\infty}$ control for switched stochastic systems with time-varying delay" by $\mathrm{G}$. Chen et al. investigates the problem of observer-based robust $H_{\infty}$ control for a class of switched stochastic systems with time-varying delay. Based on the average dwell time method, an exponential stability criterion for switched stochastic delay systems is proposed. Then, $H_{\infty}$ performance analysis and observerbased robust $H_{\infty}$ controller design for the underlying systems are developed.

"Neural network based finite-time stabilization for discretetime Markov jump nonlinear systems with time delays" by F. Chen et al. deals with the finite-time stabilization problem for discrete-time Markov jump nonlinear systems with time delays and norm-bounded exogenous disturbance. The nonlinearities in different jump modes are parameterized by neural networks. Subsequently, a linear difference inclusion state space representation for a class of neural networks is established. Based on this, sufficient conditions are derived in terms of linear matrix inequalities to guarantee stochastic finite-time stability and stochastic finite-time stabilization of the closed-loop system.

"Improved switching strategy for selective harmonic elimination in DC-AC signal generation via pulse-widthmodulation" by F. Palacios-Quiñonero et al. develops an advanced design methodology for pulse-width-modulated (PWM) DC-AC signal generation. Using design methods based on the Walsh transform, AC sinusoidal signals can be approximated by suitable PWM signals. In defining the constitutive pulses of a PWM signal, a suitable partition of the time interval is used as a reference system. In the new methodology, pulses are chosen to be symmetric with respect to the partition points, and the switching times are specified by means of switching ratios with respect to the endpoint subintervals. This approach leads to particularly simple Walsh series representations with remarkable computational simplification and achieves excellent results in reducing the harmonic distortion. For complex problems involving PWM signals with a large number of pulses, an efficient line of solution is provided by the new approach.

"Asymptotic hyperstability of a class of linear systems under impulsive controls subject to an integral popovian constraint" by M. De la Sen et al. investigates the important property of the asymptotic hyperstability of a class of continuous-time 
dynamic systems. The presence of a parallel connection of a strictly stable subsystem to an asymptotically hyperstable one in the feed-forward loop is allowed while it has also admitted the generation of a finite or infinite number of impulsive control actions which can be combined with a general form of nonimpulsive controls. The asymptotic hyperstability property is guaranteed under a set of sufficiency-type conditions for the impulsive controls.

"Delay-distribution-dependent consensus for second-order leader-follower nonlinear multiagent systems via pinning control" by $\mathrm{H}$. Li et al. studies the consensus problem for secondorder leader-follower nonlinear multiagent systems with general network topologies. A pinning control algorithm is proposed, where it includes time-varying delay information. By using the information of delay-partition and delaydistribution and constructing an appropriate LyapunovKrasovskii functional, the consensus criteria are derived to achieve leader-follower consensus for multiagent systems, which are in the form of linear inequalities that can be solved by employing the semidefinite programme method.

"On existence of stabilizing switching laws within a class of unstable linear systems" by S. S.-D. Xu and C.-C. Chen proposes a novel method to prove the equivalence of two conditions regarding the existence of stabilizing switching laws between two unstable linear systems. One is effective for theoretical derivation, while the other is implementable, and a class of stabilizing switching laws has been explicitly constructed. With the help of the equivalent relation, a condition for the existence of controllers and stabilizing switching laws between two unstabilizable linear control systems is then proposed.

"Switched convergence of second-order switched homogeneous systems" by C. Pérez and F. Benítez deals with the stabilization of second-order switched homogeneous systems. The authors establish necessary and sufficient conditions under which the stabilization is assured for a switched homogeneous system. Moreover, given an initial condition, it is shown that the method determines if there exists a switching law under which the solution converges to the origin and, if there exists this switching law, how it is constructed.

"Filippov ratio-dependent prey-predator model with threshold policy control" by X. Zhang and S. Tang investigates the sliding mode domain, sliding mode dynamics, and the existence of four types of equilibria and tangent points. Further, the stability of pseudoequilibrium is addressed by using theoretical and numerical methods, and also the local sliding bifurcations including regular/virtual equilibrium bifurcations and boundary node bifurcations are studied. Finally, some global sliding bifurcations are addressed numerically.

"Positioning errors predicting method of strapdown inertial navigation systems based on PSO-SVM" by X. Yin et al. is studied in order to evaluate the navigation errors precisely and efficiently. Firstly, the strapdown inertial navigation systems (SINS) error models that are used for error calculation are established considering several error resources with respect to inertial units. Secondly, flight paths for simulation are designed. Thirdly, the $\varepsilon$-SVR based prediction method is proposed to predict the positioning errors of navigation systems, and particle swarm optimization (PSO) is used for the support vector machine (SVM) parameters optimization. This method is also demonstrated to be effective for error prediction for an entire flight process.

Of course, the selected topics and papers do not provide an exhaustive study of all areas of this special issue. Nonetheless, they represent the rich and many-faceted knowledge that we have the pleasure of sharing with the readers.

\section{Acknowledgments}

We would like to express appreciation to the authors for their excellent contributions and patience in assisting us. The hard work of all reviewers on these papers is also very greatly acknowledged.

Chang-Hua Lien Lixian Zhang Sundarapandian Vaidyanathan Hamid Reza Karimi 


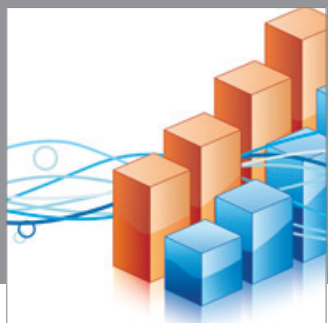

Advances in

Operations Research

mansans

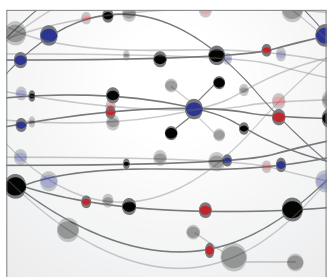

The Scientific World Journal
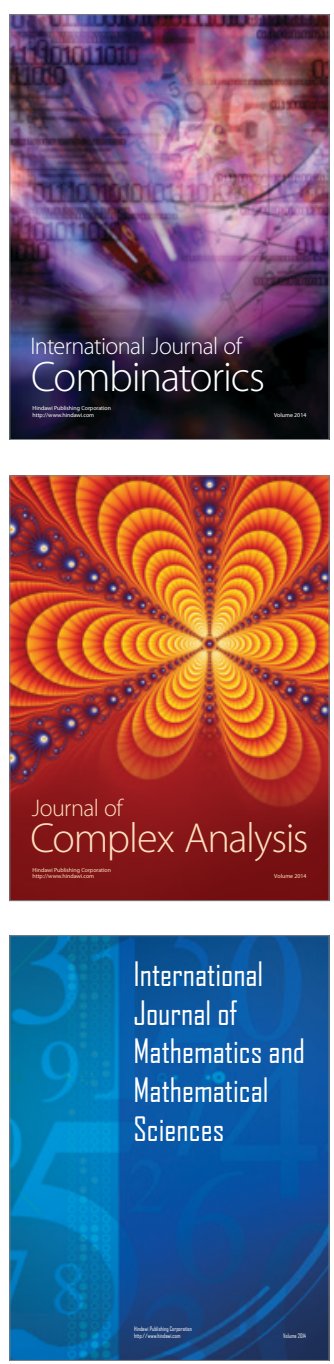
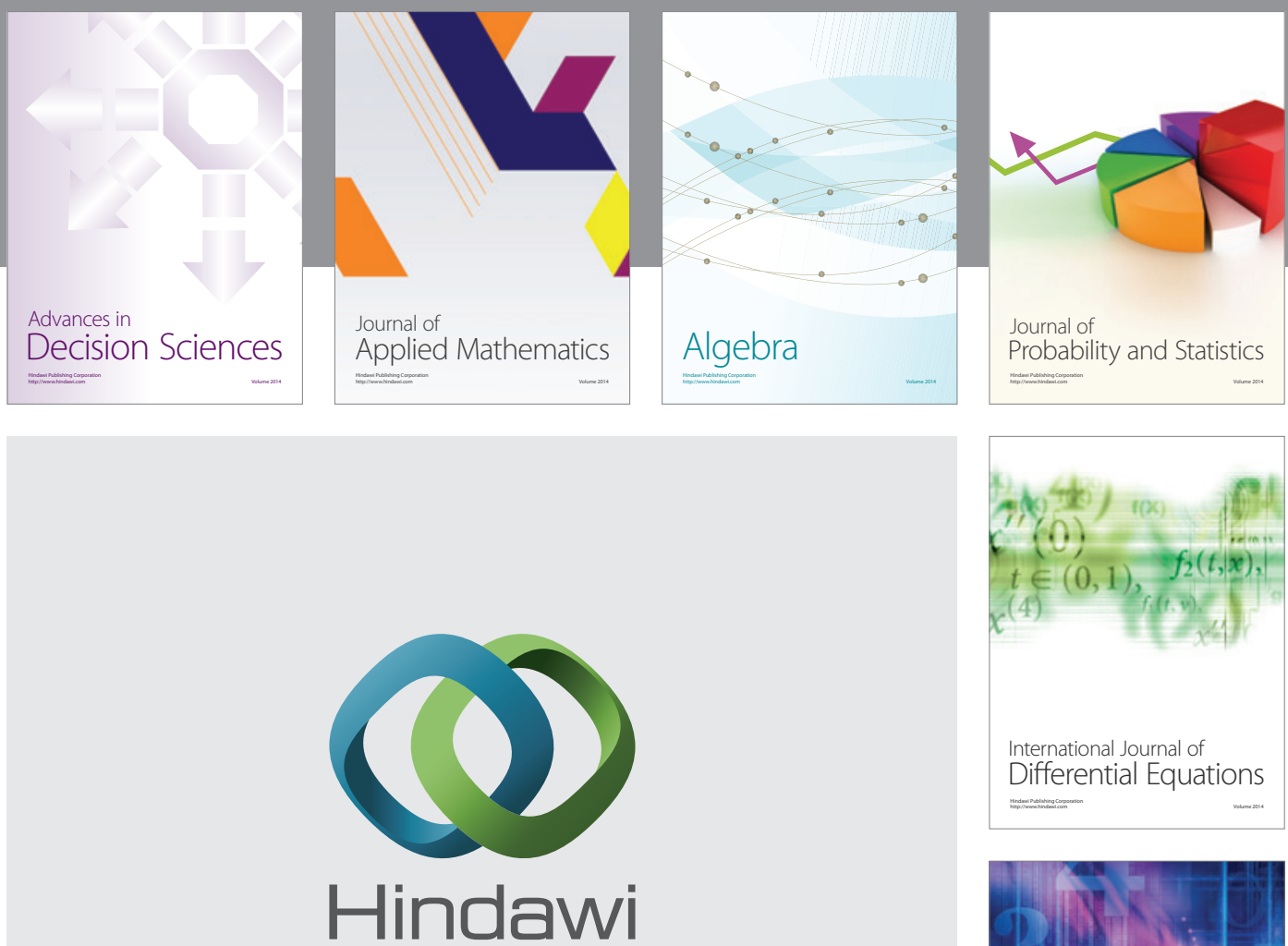

Submit your manuscripts at http://www.hindawi.com
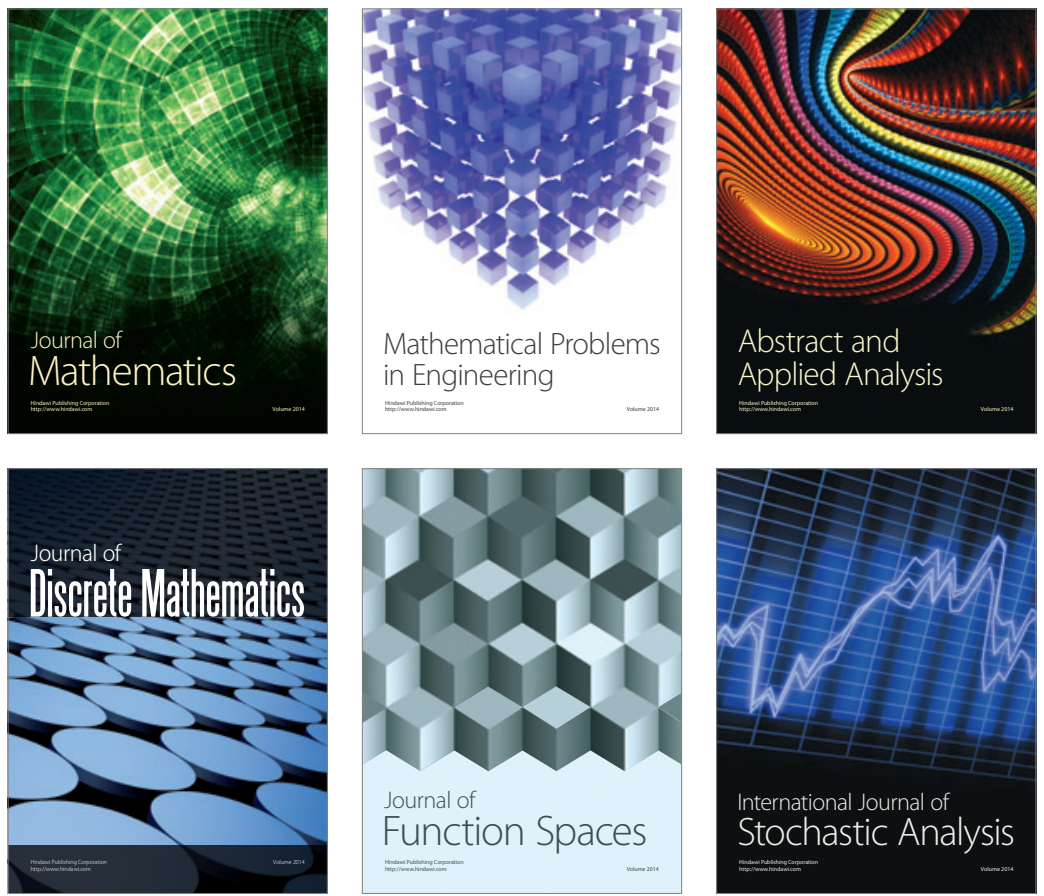

Journal of

Function Spaces

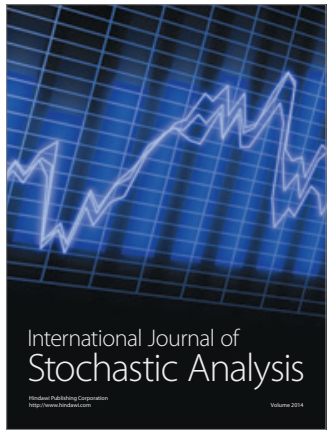

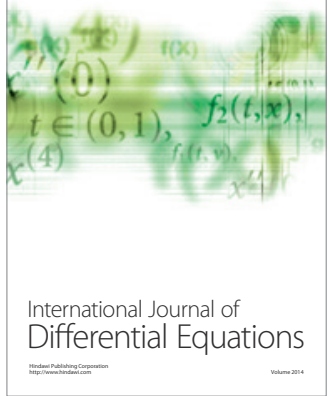
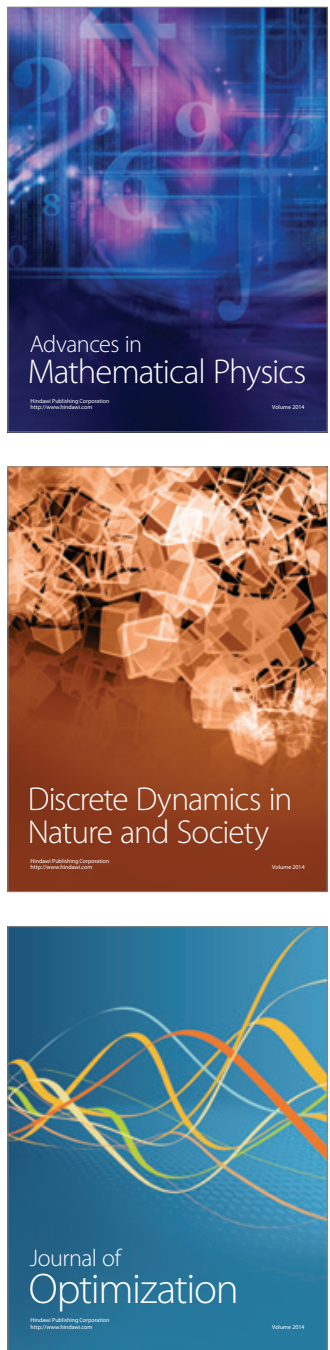